\title{
Synthesis of Potentially Bioactive Hydrazones of Pyrimidine and 1,3,5-Triazine
}

\author{
T.A. Gomktsyan, R.S. Shainova, M.V. Dovlatyan, A.P. Yengoyan* \\ Armenian National Agrarian University \\ *Russian-Armenian University \\ tiruhi.gomktsyan@mail.ru, rozetta_11@mail.ru, dovlatyan-56@mail.ru, ayengoyan@mail.ru
}

\section{A R T I CLE I N F O}

\section{Keywords:}

hydrazides,

hydrazones,

pyrimidine,

1,3,5-triazine,

plant growth stimulating activity

\begin{abstract}
A B S T RA C T
The reaction of the pyrimidine and 1,3,5-triazine hydrazides with various aldehydes formed corresponding hydrazones. Synthesized compounds are endowed with potential bioactivity. In laboratory-vegetation screening, the obtained compounds showed a pronounced stimulating effect on plant growth. The activity of the tested compounds varied in the range of $46-88 \%$ as compared to heteroauxin. Compounds that showed an activity above $70 \%$ in the experiment will be selected for deeper study and further field tests.
\end{abstract}

\section{Introduction}

Most pharmaceutical preparations that mimic biologically active natural substances consist of heterocyclic scaffolds. Hydrazides and their derivatives, in particular hydrazones, are often used as starting compounds to obtain new heterocyclic structures. Hydrazine derivatives occupy a special place in the chemotherapy of tuberculosis (Mashkovskiy, 2019). Isonicotinic acid hydrazide (isoniazid) has been used in medical practice for more than half century and has not lost its significance to this date. Based on this, ftivazide, saluside, metazide and other modified analogs with improved pharmacological properties have been obtained. In medical practice, antidepressants iproniazid and nialamide (monoamine oxidase inhibitors) are widely used (Mashkovskiy, 2019).
Studies of new hydrazine derivatives are ongoing, in which compounds with antimicrobial (Masunari and Tavaris, 2007, Loncle, et al., 2004, Küçükgüzel, et al., 2003, Vicini, et al., 2002), anti-inflammatory (Todeschini, et al., 1998), anti-tuberculosis (Bijev, 2006), antitumor (Gürsoy and Ulusoy-Güzeldemirci, 2007), antimalarial (Gemma, et al., 2006), antidepressant (Ergenç, et al., 1998) and anticonvulsant (Ragavendran, et al., 2007) activities have been revealed. Some hydrazide derivatives, particularly, herbicides (benquinox, saijunmao, phenoxyaryl hydrazides of nicotinic acid) (http://www.alanwood.net/ pesticides/) and also the compounds with plant growth stimulating activity (Gomktsyan, et al., 2012, Yengoyan, et al, 2017, Pivazyan, et al., 2019, Shainova, 2019) are used in agriculture. 


\section{Materials and methods}

We have developed an efficient method for obtaining hydrazine ( $\alpha$-methylhydrazino)-sym-triazines (Dovlatyan, et al., 1989).

Studies have shown that these compounds have herbicidal properties (Dovlatyan, et al., 1981, Dovlatyan, et al., 1980,1986) and some of their salts have fungicidal effect (Dovlatyan, 1986).

In order to expand the range of biologically active compounds, a number of compounds were interacted with aldehydes in water and appropriate hydrazones were obtained in room temperature.

\section{Results and discussions}

The targeted hydrazones, N-methylhydrazones of pyrimidine and 1,3,5-triazine were synthesized upon the reaction of the corresponding hydrazides and various aryl aldehydes in hydrochloric acid solution (Diagam). The obtained hydrazones can exist as two possible E and
$\mathrm{Z}$ structural isomers. However, according to the data of ${ }^{1} \mathrm{H}$ and ${ }^{13} \mathrm{C}$ NMR spectra, the sterically more beneficial E-isomer is predominant, and only signals of this isomer are observed in the NMR spectra. The obtained compounds were subjected to laboratory vegetation tests to determine herbicidal, fungicidal, growth-regulating properties. Almost all of the compounds obtained showed a stimulating effect on plant growth. The experiments were carried out on seeds and seedlings of common beans (Phaseolus vulgaris L.). The effect of aqueous suspensions of compounds at the concentrations of 25 and $50 \mathrm{mg} / \mathrm{L}$ on seed viability, germination, and seedling growth was studied. These data were compared with the effect of heteroauxin solutions of the same concentrations. The activity of the tested compounds varied in the range of $46-88 \%$ as compared to heteroauxin. In some cases, the growth-stimulating effect of solutions with a lower concentration turned out to be stronger than that of more concentrated solutions. Compounds that showed an activity above $70 \%$ in the experiment will be selected for deeper study and further field tests using their solutions with concentrations less than $25 \mathrm{mg} / \mathrm{L}$.

$$
\text { , } 3 \mathrm{X}=\mathrm{CH} ; 2,4 \mathrm{X}=\mathrm{N}
$$<smiles>[R]c1nc([R])nc(NNC=[Y19])n1</smiles>

$\mathrm{R}=s-\mathrm{C}_{4} \mathrm{H}_{9} \mathrm{NH} ; t-\mathrm{C}_{4} \mathrm{H}_{9} \mathrm{NH} ; \mathrm{C}_{2} \mathrm{H}_{5} \mathrm{NH} ;\left(\mathrm{CH}_{3}\right)_{2} \mathrm{~N}, \mathrm{CH}_{3} \quad \mathrm{R}_{1}=\mathrm{OCH}_{3} ; \mathrm{CH}_{3} \mathrm{NH} ; \mathrm{N}\left(\mathrm{CH}_{3}\right)_{2}$<smiles>Cc1cccc([N+](=O)c2ccc(C)cc2OCc2ccc(Cc3ccc4c(c3)OCO4)c(O)c2)c1</smiles><smiles>[R]c1nc([R])nc(N(C)N)n1</smiles><smiles>[R]c1nc([R])nc(N(C)/N=C/[Al])n1</smiles>

$\mathrm{R}=i-\mathrm{C}_{3} \mathrm{H}_{7} \mathrm{NH} ;\left(\mathrm{CH}_{3}\right)_{2} \mathrm{~N} ; \mathrm{C}_{2} \mathrm{H}_{5} \mathrm{NH} \quad \mathrm{R}_{1}=\mathrm{OCH}_{3} ; \mathrm{SCH}_{3} \quad \mathrm{Ar}=\overbrace{\mathrm{OCH}_{3}}^{\mathrm{OH}}$

Diagram. Synthesis of hydrazones, N-methylhydrazones of pyrimidine and 1,3,5-triazine (composed by the authors). 


\section{Experimental}

${ }^{1} \mathrm{H}$ and ${ }^{13} \mathrm{C}$ NMR spectra were recorded at $30^{\circ} \mathrm{C}$ on a Varian Mercury-300 NMR spectrometer (300 and $75 \mathrm{MHz}$, respectively) in a mixture of solvents CC14-DMSO-d6 (3:1); TMS was used as an internal standard. The reaction progress and the purity of the obtained compounds were monitored by TLC on Silufol UV-254 plates; benzenehexane, 2:1, mixture was used as an eluent. Elemental analysis was performed on a Eurovector EA3000 CHNS analyzer. Melting points were determined by the capillary method and were not corrected.

\section{General procedure}

To the mixture of compounds $1,2,5$ or $7(10 \mathrm{mmol}), 10 \mathrm{~mL}$ of water and $1,5 \mathrm{~mL}$ of $\mathrm{HCl}(36 \%), 10 \mathrm{mmol}$ of various aldehydes were added and the reaction mixture was stirred at $20{ }^{\circ} \mathrm{C}$ for $24 \mathrm{~h}$. After adding water $(10-15 \mathrm{~mL})$ the precipitate was filtered off and dried.

\section{Compounds examples}

(E)-4-(2-benzylidenehydrazinyl)-2-amino-6-methylpyrimidine:

Compound yield - $82 \%$, m.p. $255^{\circ} \mathrm{C}$ (decomp). ${ }^{1} \mathrm{H}$ NMR, $\delta$ : 2.40 (s, 3H, $\left.\mathrm{CH}_{3}\right) ; 6.61$ (s, 1H, CH-pyrim.); 7.34-7.72 (m, 5H, $\left.\mathrm{C}_{6} \mathrm{H}_{5}\right) ; 7.80$ (br.s, $\left.2 \mathrm{H}, \mathrm{NH}_{2}\right) ; 8.31(\mathrm{~s}, 1 \mathrm{H}, \mathrm{CH}=\mathrm{N})$; 13.54 (br.s, $1 \mathrm{H}, \mathrm{NH}) .{ }^{13} \mathrm{C}$ NMR, $\delta: 18.4,95.5,126.9$, 128.1, 129.6, 133.6, 147.1, 153.6, 155.5, 162.7. Anal.calc.: Found: C, 63.29; H, 5.67; N, 30.93. $\mathrm{C}_{12} \mathrm{H}_{13} \mathrm{~N}_{5}$. Calcul.: C, 63.42; H, 5.77; N, 30.82.

(E)-4-(2-(4-methoxybenzylidene)hydrazinyl)-2-amino-6methylpyrimidine:

Compound yield - $88 \%$, m.p. $170-172{ }^{\circ} \mathrm{C}$. ${ }^{1} \mathrm{H}$ NMR, $\delta$ : $2.26\left(\mathrm{~s}, 3 \mathrm{H}, \mathrm{CH}_{3}\right) ; 3.82\left(\mathrm{~s}, 3 \mathrm{H}, \mathrm{OCH}_{3}\right) ; 6.36$ (br.s, $2 \mathrm{H}$, $\mathrm{NH}_{2}$ ); 6.41 (s, 1H, CH-pyrim.); 6.85-7.61 (m, 4H, $\left.\mathrm{C}_{6} \mathrm{H}_{4}\right)$; 8.02 (s, 1H, CH=N); 11.04 (br.s, $1 \mathrm{H}, \mathrm{NH}) .{ }^{13} \mathrm{C}$ NMR, $\delta$ : 21.6, 54.6, 92.3, 113.5, 127.1, 127.7, 142.8, 159.8, 160.1, 161.0, 162.3. Anal.calc.: Found: C, 60.74; H, 5.95; N, 27.40. $\mathrm{C}_{13} \mathrm{H}_{15} \mathrm{~N}_{5} \mathrm{O}$. Calcul.: C, 60.69; H, 5.88; N, 27.22.

(E)-4-(2-(furan-2-ylmethylene)hydrazinyl)-2-amino-6methylpyrimidin:

Compound yield - 83\%, m.p. $260{ }^{\circ} \mathrm{C}$ (decomp). ${ }^{1} \mathrm{H}$ NMR, $\delta$ : $2.39\left(\mathrm{~s}, 3 \mathrm{H}, \mathrm{CH}_{3}\right) ; 6.52-6.54\left(\mathrm{~d} \times \mathrm{d}, \mathrm{J}_{1}=3.4 \mathrm{~Hz}, \mathrm{~J}_{2}=1.8\right.$ $\mathrm{Hz}, 2 \mathrm{H}, \mathrm{CH}=\mathrm{CH}$ fur.); 6.81 (d, $\mathrm{J}_{1}=3.4 \mathrm{~Hz}, 1 \mathrm{H}, \mathrm{CH}-$ fur.); 7.64 (s, 1H, CH-pyrim.); 7.76 (br.s, $2 \mathrm{H}, \mathrm{NH}_{2}$ ); 8.19 (s, 1H, $\mathrm{CH}=\mathrm{N}$ ); 12.20 (br.s, $1 \mathrm{H}, \mathrm{NH}) .{ }^{13} \mathrm{C} \mathrm{NMR}, \delta: 18.4,93.0$, 111.6, 113.0, 136.8, 144.3, 149.0, 153.6, 155.4, 162.5 . Anal.calc.: Found: C, 55.37; H, 5.02; N, 32.43. $\mathrm{C}_{10} \mathrm{H}_{11} \mathrm{~N}_{5} \mathrm{O}$. Calcul.: C, 55.29; H, 5.10; N, 32.24;
(E)-4-(2-benzylidenehydrazinyl)-2-amino-6-methyl-1,3,5triazine:

Compound yield - $81 \%$, m.p. $280-282{ }^{\circ} \mathrm{C} .{ }^{1} \mathrm{H}$ NMR, $\delta$ : $2.28\left(\mathrm{~s}, 3 \mathrm{H}, \mathrm{CH}_{3}\right) ; 6.80$ (br.s, $\left.2 \mathrm{H}, \mathrm{NH}_{2}\right) ; 7.25-7.73(\mathrm{~m}$, $\left.5 \mathrm{H}, \mathrm{C}_{6} \mathrm{H}_{5}\right) ; 8.10(\mathrm{~s}, 1 \mathrm{H}, \mathrm{CH}=\mathrm{N}) ; 11.08$ (br.s, $\left.1 \mathrm{H}, \mathrm{NH}\right) .{ }^{13} \mathrm{C}$ NMR, $\delta: 24.0,126.4,127.9,128.4,134.7,143.4,173.5$. Anal.calc.: Found: C, 57.96; H, 5.25; N, 36.96. $\mathrm{C}_{11} \mathrm{H}_{12} \mathrm{~N}_{6}$. Calcul.: C, 57.88; H, 5.30; N, 36.82 .

(E)-4-(2-(4-methoxybenzylidene)hydrazinyl)-2-amino-6methyl-1,3,5-triazine:

Compound yield - 83\%, m.p. $248-250{ }^{\circ} \mathrm{C} .{ }^{1} \mathrm{H}$ NMR, $\delta: 2.20$ (s, 3H, $\left.\mathrm{CH}_{3}\right) ; 3.81$ (s, 3H, $\left.\mathrm{OCH}_{3}\right) ; 6.50$ and 7.17 (br.s, $2 \mathrm{H}$, $\left.\mathrm{NH}_{2}\right) ; 6.84-7.63\left(\mathrm{~m}, 4 \mathrm{H}, \mathrm{C}_{6} \mathrm{H}_{4}\right) ; 8.00(\mathrm{~s}, 1 \mathrm{H}, \mathrm{CH}=\mathrm{N}) ; 10.74$ (br.s, $1 \mathrm{H}, \mathrm{NH}) .{ }^{13} \mathrm{C} \mathrm{NMR}, \delta: 24.4,54.7,113.7,127.4$, 127.7, 128.1, 142.7, 159.8, 163.7; 166.5. Anal.calc.: Found: C, 55.72; H, 5.38; N, 32.38. $\mathrm{C}_{12} \mathrm{H}_{14} \mathrm{~N}_{6} \mathrm{O}$. Calcul.: C, 55.80; H, 5.46; N, 32.54 .

(E)-4-((2-(4-(ethylamino)-6-methoxy-1,3,5-triazin-2-yl)2-methylhydrazono)methyl)-2-methoxyphenol:

Compound yield - 67\%, m.p. 203-205 ${ }^{\circ} \mathrm{C} .{ }^{1} \mathrm{H}$ NMR, $\delta: 1.29$ (t, J=7.2, 3H, $\left.\mathrm{NCH}_{2} \underline{\mathrm{CH}}_{3}\right) ; 3.50$ (q, J=7.2, $2 \mathrm{H}, \mathrm{NCH}_{2} \mathrm{CH}_{3}$ ); $3.64\left(\mathrm{~s}, 3 \mathrm{H}, \mathrm{NCH}_{3}\right) ; 3.98\left(\mathrm{~s}, 3 \mathrm{H}, \mathrm{OCH}_{3}\right) ; 4.00(\mathrm{~s}, 3 \mathrm{H}$, $\left.\mathrm{OCH}_{3}\right) ; 6.75-7.97\left(\mathrm{~m}, 3 \mathrm{H}, \mathrm{C}_{6} \mathrm{H}_{3}\right) ; 8.14(\mathrm{~s}, 1 \mathrm{H}, \mathrm{CH}=\mathrm{N})$; 10.62 (br.s, $1 \mathrm{H}, \mathrm{OH}) .{ }^{13} \mathrm{C} \mathrm{NMR}, \delta: 13.7,30.2,35.5,55.2$, 56.5, 110.7, 114.5, 124.1, 124.8, 148.0, 148.1, 155.1, 157.2, 168.4. Anal.calc.: Found: C, 54.14; H, 6.01; N, 25.41. $\mathrm{C}_{15} \mathrm{H}_{20} \mathrm{~N}_{6} \mathrm{O}_{3}$. Calcul.: C, 54.21; H, 6.07; N, 25.29.

\section{Conclusion}

Based on the aforestated methods and examples, it can be summed up that a series of pyrimidine and 1,3,5-triazine hydrazides derivatives were synthesized, which, upon biological screening, showed a pronounced stimulating effect on plant growth. The most active ones have been selected for deeper research and subsequent field trials.

\section{References}

1. Bijev, A. (2006). New Heterocyclic Hydrazones in the Search for Antitubercular Agents: Synthesis and in Vitro Evaluations // Lett. Drug Des. Discovery, - vol. 3, - pp. 506-512. https://doi.org/10.2174/1570180067 78194790 .

2. Dovlatyan, V.V., Gomktsyan, T. A. and Khachatryan, N. Kh. (1989). Synthesis of $\alpha$ - Methylhydrazinosimm-triazines // Khim. Zh. Arm., - vol. 42, - N 3, - pp. 187-191. 
3. Dovlatyan, V.V., Khachatryan, N. Kh., Gomktsyan, T. A., Andreeva, E. I., Smirnova, K. F. and Filimonova, L. A. (1980). Rhodonite Salts of $\alpha$-Methylhydrazino-symtriazines Derivatives with Fungicidal Activity // Patent no. 799332 .

4. Dovlatyan, V.V., Khachatryan, N. Kh., Gomktsyan, T. A., Berezovski, M.Y., Salamatina, N.I. and Usacheva, N.M. (1981). Derivatives of $\alpha$-Methylhydrazino-sym-triazines Showing Herbicidal Activity // Patent no. 904294.

5. Dovlatyan, V.V., Khachatryan, N. Kh., Gomktsyan, T. A., Berezovski, M.Y., Minaev, L.I., Balesta, P. S, Derevko, V. I., Kolcova, S. S. and Miheev, V. M. (1986). Derivatives of $\alpha$-Methylhydrazino-symtriazines Showing Herbicidal Activity // Patent no. 1293982.

6. Ergenç, N., Günay, N.S., and Demirdamar, R. (1998). Synthesis and Antidepressant Evaluation of New 3-Phenyl-5Sulfonamidoindole Derivatives // Eur. J. Med. Chem., - vol. 33, - pp. 143-148. https://doi. org/10.1016/S0223-5234(98)80039-1.

7. Gemma, S., Kukreja, G., Fattorusso, C., Persico, M., Romano, M., Altarelli, M., Savini, L., Campiani, G., Fattorusso, E., and Basilico, N. (2006). Synthesis of N1-Arylidene-N2-Quinolyl- and N2Acrydinylhydrazones as Potent Antimalarial Active Agents against CQ-Resistant P. falciparum Strains // Bioorg. Med. Chem. Lett., - vol. 16, - pp. 5384-5388. https://doi.org/10.1016/j.bmcl.2006.07.060.

8. Gomktsyan, T.A., Karapetyan, A.V., Abrahamyan, L.G., and Yengoyan, A.P. (2012). Synthesis of Substituted Acylhydrazine-1,3,5-triazine // Khim. Zh. Arm., - vol. 65, - N 3, - pp. 342-348.

9. Gürsoy, E., and Ulusoy-Güzeldemirci, N. (2007). Synthesis and Primary Cytotoxicity Evaluation of New Imidazo[2,1-b]Thiazole Derivatives // Eur. J. Med. Chem., - vol. 42, - pp. 320-326. https://doi. org/10.1016/j.ejmech.2006.10.012.

10. http://www.alanwood.net/pesticides/class pesticides. html (accessed on 18.11.2021).

11. Küçükgüzel, S.G., Mazi, A., Sahin, F., Öztürk, S., and Stables, J.P. (2003). Synthesis and Biological Activities of Diflunisal Hydrazide /Hydrazones // Eur. J. Med. Chem., - vol. 38, - pp. 1005-1013. https://doi. org/10.1016/j.ejmech.2003.08.004.

12. Loncle, C., Brunel, J., Vidal, N., Dherbomez, M., and Letourneux, Y. (2004). Synthesis and Antifungal Activity of Cholesterol-Hydrazone Derivatives // Eur.
J. Med. Chem, -vol. 39, - pp. 1067-1071. https://doi. org/10.1016/j.ejmech.2004.07.005.

13. Mashkovskiy, M.D. (2019). // Lekarstvennye Sredstva (Drugs), Moscow: Novaya Volna.

14. Masunari, A., and Tavaris, L.C. (2007). A new Class of Nifuroxazide Analogues: Synthesis of 5-Nitrothiophene Derivatives with Antimicrobial Activity against Multidrug-Resistant Staphylococcus Aureus // Bioorg. Med. Chem., - vol. 15, - pp. 42294236. https://doi.org/10.1016/j.bmc.2007.03.068.

15. Pivazyan, V.A., Ghazaryan, E.A., Azaryan, Zh.A., and Yengoyan, A.P. (2019). Synthesis and Biological Activity of New 2-\{[6-Methyl-2-(Pyrrolidin-1-yl) Pyrimidin4-yl]Oxy\} Acetohydrazide Derivatives // Russ. J. Gen. Chem., - vol. 89, - N 10, - pp. 2010-2017. https://doi.org/10.1134/S1070363219100050.

16. 16. Ragavendran, J., Sriram, D., Patel, S., Reddy, I., Bharathwajan, N., Stables, J., and Yogeeswari, P. (2007). Design and Synthesis of Anticonvulsants from a Combined PhthalimideeGABAeanilide and Hydrazone Pharmacophore // Eur. J. Med. Chem., - vol. 42, - pp. 146-151. https://doi.org/10.1016/j. ejmech.2006.08.010.

17. Shainova, R.S., Gomktsyan, T.A., Karapetyan, A.V., and Yengoyan, A.P. (2019). Synthesis and Biological Evaluation of 3-O-Substituted 1-Benzyl6-Oxo-1,6-Dihydropyridazine Derivatives // J. Chem. Res., - vol. 43, - N 9-10, - p. 352. https://doi. org/10.1177/1747519819866402.

18. Todeschini, A.R., Miranda, A.L., Silva, C.M., Parrini, S.C., and Barreiro, E.J. (1998). Synthesis and Evaluation of Analgesic, Anti-Inflammatory and Antiplatelet Properties of New 2-Pyridylarylhydrazone Derivatives // Eur. J. Med. Chem., - vol. 33, - pp. 189199. https://doi.org/10.1016/S0223-5234(98)80008-1.

19. Vicini, P., Zani, F., Cozzini, P., and Doytchinova, I. (2002). Hydrazones of 1,2-Benzisothiazole Hydrazides: Synthesis, Antimicrobial Activity and QSAR Investigations // Eur. J. Med. Chem., - vol. 37, - pp. 553-564. https://doi.org/10.1016/S02235234(02)01378-8.

20. Yengoyan, A.P., Gomktsyan, T.A., Karapetyan, A.V., and Shainova, R.S. (2017). Comparison of Traditional Methods with the Method of MW Radiation in the Synthesis of New Biologically Active Pyridazine Derivatives // Chemical Journal of Armenia, - vol. 70, N 1-2, - pp. 199-217. 